\title{
Systematischer Überblick über das Hörsturzklientel - eine Mixtur aus Patienten mit und ohne Perilymphfisteln
}

\subsection{Einleitung}

\subsubsection{Hinführung zum Thema}

Plötzlich aufgetretene Perilymphfisteln (PLF) unterscheiden sich phänomenologisch oft nicht vom sog. Hörsturz. Neben der akut eingetretenen Hörminderung, die in der Regel sehr ausgeprägt ist - es kommt zum Abfließen von Perilymphe aus dem Innenohr -, tritt oft zeitgleich mit dem Hörverlust ein heftiger Schwindel auf, gelegentlich zusätzlich begleitet von Tinnitus auf dem betroffenen $\mathrm{Ohr}[3,6$, $9,12,22-25,29-31,37,38,43,57,59,61,65,75,78,84,87,89,97,104]$.

\subsubsection{Pathophysiologie und Diagnostik der PLF}

Eine sichere, präoperative, diagnostische Identifizierung der PLF ist trotz Beschreibung verschiedener Methoden, die mit unterschiedlichen Verfahrensansätzen zur Lösung des Problems beitragen wollen, bislang nicht zufriedenstellend möglich $[2,4,8,36,39,50,70,83,105]$. Demzufolge wird der Verdacht auf eine solche Schädigung meist durch das Bestehen einer Konstellation typischer, seitens der Patienten geschilderter, anamnestischer Beschwerden und dem begleitenden Vorliegen charakteristischer Ausgangsbefunde erhoben.

Pathophysiologisch wird bei der PLF entweder eine plötzliche Erhöhung des intrazerebralen Druckes oder ein akuter Anstieg des Mittelohrdruckes mit der Entstehung eines Lecks zwischen dem Innen- und Mittelohr in Verbindung gesetzt (in- und explosive Ursache der Membranruptur) [3, 6, 9, 10, 12, 22-24, 28-31, $42,43,52,53,56,57,61,65,75,78,84,87-89,96,97,99,101]$. Derartige 
Situationen können u. a. mit einer Erhöhung des intraabdominellen Druckes infolge Aktivierung der Bauchpresse bei starker physischer Anstrengung, plötzlichen Nies- oder Hustenattacken im Rahmen eines Infektes der oberen Atemwege, heftigem Würgen, aber auch mit Barotraumen verbunden sein. Auch direkte Kopfverletzungen können mit einem direkten Einreißen der Membranen, insbesondere am runden Fenster, einhergehen.

\subsubsection{Physiologische Erklärung der Wirkung einer Abdeckung der runden/ovalen Fenstermembran bei Patienten ohne pathologische Mittelohrbefunde}

Als physiologische Erklärung, warum eine Tympanoskopie mit Obliteration eines oder beider Fenster auch bei einem nicht als PLF anmutenden Hörsturz in einem hohen Prozentsatz der Fälle zu einer Hörverbesserung beigetragen hat, wird in der Literatur angeführt, dass es einerseits durch die Traumatisierung der Pauke im Rahmen des chirurgischen Eingriffs und andererseits durch das Einbringen von ,totem“ Bindegewebe, das als Entzündungsreiz fungiert, zu einer Hyperämie im Bereich der basalen Kochleawindung mit gehörverbesserndem Effekt kommt [42]. Zusätzlich wurde in Tierversuchen nachgewiesen, dass es in einem Zeitraum von 14 Tagen nach Zerstörung der Rundfenstermembran „spontan“ wieder zur Generierung von Hörpotenzialen in der Schnecke kam, es also zu einem naturgegebenen Verschluss des Lecks mit anschließender Wiederauffüllung der Perilymphräume gekommen sein muss [42].

\subsubsection{Fragestellung}

In diesem Kapitel wird anhand des eigenen Patientengutes (Patienten mit pathologischer Mittelohrstruktur: $\mathrm{n}=7$ [IOP-Positive]; Patienten ohne Paukenauffälligkeit: $\mathrm{n}=44$ [IOP-Negative]) sowie anhand von 4 Studienkollektiven $(n=317)$ aus der Literatur untersucht, inwiefern sich das Krankheitsbild des Hörsturzes von dem der PLF abgrenzen lässt, ob sich das zu den beiden Erkrankungsentitäten gehörende Patientengut hinsichtlich bestimmter Merkmale grundsätzlich voneinander unterscheidet und welche Wirkung die Abdichtung der runden/ovalen Fensternische auf die weitere Hörentwicklung in beiden Populationen nimmt [29, 30, 33, 57, 75, 78, 97]. Unser eigenes Patientengut haben wir, wo es methodisch notwendig war, dabei jeweils den Auswertungsmodalitäten der einzelnen Autoren angepasst, die auch über dieses Thema referiert haben [29, 75]. Nicht zuletzt soll der Frage nachgegangen werden, mit welcher Sicherheit präoperativ eine Prognose über das Bestehen von Mittelohrauffälligkeiten gestellt werden kann. 


\subsection{Ergebnisse}

\subsubsection{Aussagekraft von sog. Auslösesituationen und Schwindel bezüglich der Vorhersage einer PLF}

\subsubsection{Eigenes Patientengut}

\subsection{Rekrutierung des eigenen Patientengutes}

Insgesamt haben wir bei $7(n=9,2 \%)$ der 76 tympanoskopierten Patienten pathologische Mittelohrbefunde finden können. Dreimal bestand eine Flüssigkeitsansammlung an der runden Fensternische, 3-mal eine knöcherne Läsion/ Fissuren im Promontoriumbereich und 1-mal eine Stapesfußplattenfraktur mit Abtropfen von Perilymphe aus dem ovalen Fenster. Es zeigte sich bei den IOPPositiven also ein uneinheitliches pathologisch-anatomisches Bild. Folgt man den Angaben von Lamm et al., kann auch bei Patienten, die lediglich Fissuren in der Kochleawand aufweisen, durchaus die Möglichkeit bestehen, dass über diese feinen Haarrisse im Laufe der Zeit Perilymphe abgeflossen ist [42].

Bei einem der 7 Patienten aus unserem Patientenkollektiv betrug der mittlere Hörverlust $<60 \mathrm{~dB}$. Dieser Patient erschien auch erst weit mehr als 1 Woche (58 Tage) nach dem Hörsturzereignis in der Klinik. Er wurde deshalb in der nachfolgenden Auswertung, zumindest was die Hörerfolge angeht, nicht berücksichtigt. Von zwei weiteren Patienten konnten wir lediglich die bei Krankenhausaufnahme erhobenen anamnestischen Daten und klinischen Befunde verwerten, für die weitere Verlaufsbeobachtungen fehlten die audiometrischen Messdaten. Damit konnten wir lediglich ein bescheiden kleines Patientengut zur Gesamtanalyse beisteuern $(n=4)$.

Die Spanne, innerhalb derer in der Literatur über die Aufdeckung von Perilymphfisteln im Rahmen explorativer Tympanoskopien bei hochgradigen akuten Hörverlusten berichtet wird, reicht nach Literaturmitteilungen von $0 \%$ bis nahe $80 \%$ [38, 65, 97]. Eine Deutung dieser großen Variationsbreite kann auch dahin gehend erfolgen, dass selbst die Paukeninspektion kein absolut valides Ergebnis liefert und damit nicht unbedingt als „Goldstandard“ eingesetzt werden kann. In der Bewertung haben möglicherweise auch die für eine PLF charakteristischen Angaben bezüglich des Vorliegens einer typischen Auslösesituation und die Symptomschilderungen der Patienten, besonders bezogen auf den zeitgleich mit dem Hörsturz aufgetretenen Schwindel, einen höheren Stellenwert, als man gemeinhin glaubt. Eine weitere Erklärung der großen Spannbreite kann darüber hinaus in der Verschiedenartigkeit der Kriterien liegen, von denen die Operateure ihre Entscheidung, welche intraoperativen Beobachtungen sie mit der Aussage „PLF vorhanden oder nicht“ verknüpft haben. Auch werden die Indikationskriterien für die Durchführung der Tympanoskopie zwischen den einzelnen Autoren variiert haben, sodass sie Patienten mit verschieden hohen Vorhersagewahrscheinlichkeiten für das Bestehen eines Innen-/Mittelohrlecks in ihren Vergleich einbezogen haben. 


\subsection{Verteilung der Häufung von Merkmalsunterschieden}

Bei dem Vergleich, wie die beiden Subpopulationen - IOP-Negative und IOPPositive - zusammengestellt sind, erkennt man aus der Tab. 7.1, dass sich die beiden Gruppen in unserem Patientengut in keinem der untersuchten Merkmale signifikant unterschieden.

\section{Hintergrundinformation}

Lediglich hinsichtlich des Vorkommens von Schwindel $(\mathrm{p}=0,05)$ bestanden im ersten Schritt der Auswertung statistisch relevante Unterschiede in der Häufigkeitsverteilung zwischen den IOP-positiven und IOP-negativen Patienten derart, dass die Erkrankten mit Mittelohrpathologien wesentlich häufiger (57\%) Gleichgewichtsstörungen aufwiesen als diejenigen, die normale Paukenstrukturen zeigten (26,4\%). Dieses Ergebnis hielt aber der Post-hoc-Analyse mit der Bonferroni- wie auch der Li-Korrektur einer zugrunde gelegten 5-\%-Irrtumswahrscheinlichkeit nicht mehr stand, fand auch keine Bestätigung im Kruskal-Wallis-Test.

Vielleicht hatten bei einem Teil dieser Patienten nicht realisierte, frühere Traumen vorgelegen, die von ihnen evtl. als Hörstürze, aber ohne erkennbare Ätiologie eingeordnet worden waren (möglicherweise bei 2 Patienten). Bei der traumatischen Trommelfellperforation kann die Beziehung zu einem früheren Unfall sogar direkt hergestellt werden, wobei die Ohrschädigung in diesem Fall möglicherweise über die Trommelfellebene hinaus gegangen war und auch knöcherne/membranöse Strukturen des Mittelohres mit erfasst hatte. Es scheint also ratsam, sich im Rahmen der Hörsturzanamnese detailliert über frühere Kopf-/Ohrtraumen zu orientieren.

\subsection{Mittelohrbefunde und Auslösesituation}

Eine für eine PLF typische Auslösesituation wurde von 16/52 unserer Patienten (IOP-Positive und IOP-Negative; 27,6 \%) angegeben (Tab. 7.2). Nur in 2 Fällen fanden sich dabei pathologische Mittelohrbefunde (1-mal Flüssigkeitsansammlung in der runden Fensternische nach Hustenattacke, 1-mal Stapesfraktur nach

Tab. 7.1 Wahrscheinlichkeiten, mit denen sich IOP-positive und IOP-negative Patienten hinsichtlich verschiedener Parameter unterschieden

\begin{tabular}{l|l|l|l}
\hline & Wahrscheinlichkeit $(\mathrm{p})$ & Nach Bonferroni-Korrektur & Nach Li-Korrektur \\
\hline Geschlecht & 0,26 & & \\
\hline Vorerkrankung & 0,07 & & \\
\hline Vortherapie & 0,33 & & \\
\hline Auslöser & 0,92 & & \\
\hline Schwindel & $0,05^{*}$ & 0,55 & 0,59 \\
\hline Tinnitus & 0,36 & & \\
\hline Seite & 0,28 & & \\
\hline Alter & 0,87 & & \\
\hline A-HV & 0,63 & & \\
\hline Hörgewinn & 0,3 & & \\
\hline End-HV & 0,25 & & \\
\hline
\end{tabular}

Vorerkrankung $=$ Innenohrvorerkrankung/SHT; Vortherapie $=$ ambulante Vortherapien; Anfangs$H V=$ Anfangshörverlust; End- $H V=$ Endhörverlust; ${ }^{*}=$ signifikant 
Tab. 7.2 Zusammenhänge zwischen IOP-positiven und IOP-negativen Befunden und dem Vorliegen eines für die PLF typischen Auslösers im eigenen Patientengut (oberer Tabellenteil), im Patientengut von Maier et al. (mittlerer Tabellenteil) und im zusammengefassten Patientengut (unterer Tabellenteil)

\begin{tabular}{l|l|l}
\hline Eigenes Patientengut & IOP path. & IOP o. B. \\
\hline & 2 & 14 \\
\hline Auslöser+ & 4 & 38 \\
\hline Auslöser- & &
\end{tabular}

Beziehung: Intratympanaler Befund - Auslöser

OR $=1,05 \%$

Prädiktiver Wert: $12,5 \%$

Sensitivität: $33,3 \%$

Spezifität: $73,1 \%$

$p=0,77$

Patientengut von Maier et al. [57]

\begin{tabular}{l|l|l}
\hline & IOP path. & IOP o. B. \\
\hline Auslöser+ & 52 & 24 \\
\hline Auslöser - & 31 & 28 \\
\hline
\end{tabular}

Beziehung: Intratympanaler Befund - Auslöser

OR $=1,96$

Prädiktiver Wert: 68,4\%

Sensitivität: $61,9 \%$

Spezifität: $53,8 \%$

$p=0,059$

Aus beiden Studien zusammengefasstes Patientengut

\begin{tabular}{l|l|l}
\hline & IOP path. & IOP o. B. \\
\hline Auslöser + & 54 & 38 \\
\hline Auslöser - & 35 & 66 \\
\hline
\end{tabular}

Beziehung: Intratympanaler Befund - Auslöser

OR $=1,9$

Prädiktiver Wert: $30,2 \%$

Sensitivität: $59,3 \%$

Spezifität: $63,5 \%$

$p=0,0008$

IOP path.=Patienten mit pathologischen Mittelohrstrukturen; IOP $o . \quad B .=$ Patienten mit normalen Mittelohrstrukturen; $O R=$ Odds Ratio; $p=$ Wahrscheinlichkeitswert, mit der sich beide Subgruppen von Patienten unterschieden

Alkoholexzess). Umgekehrt ging laut Patientenschilderung das Hörsturzereignis 42-mal ohne Bestehen einer solchen Auslösesituation einher (72,4\%; prädiktiver Wert: $12,5 \%$ ). Diese verteilten sich auf 4 Patienten mit auffälligen Mittelohrbefunden und 38 Patienten mit regelrechten Paukenstrukturen. Die Odds-Ratio betrug 1,05 und die Wahrscheinlichkeit, dass sich die beiden Populationen (IOP-Positive und -Negative) hinsichtlich der Häufigkeit des Auftretens einer typischen Auslösesituation unterschieden lag bei 0,77. 
Eine vergleichbare Auswertung der Patientendaten liegt lediglich von Maier et al. vor (Tab. 7.2) [57]. Sie konnten einen engeren Zusammenhang zwischen dem Vorliegen einer typischen Auslösesituation und den intraoperativ eruierten Paukenbefunden herstellen (prädiktiver Wert: 68,4\%; $\mathrm{p}=0,059$ ) als wir.

In dem aus beiden Studien zusammengefassten Datenmaterial konnte ein prädiktiver Wert von 30,2\% und eine Odds Ratio von 1,9 ermittelt werden. Die Wahrscheinlichkeit dafür, dass eine statistische unterschiedliche Verteilung in der Häufigkeit des Vorliegens eines typischen Auslösers und in der Gruppe der IOPPositiven und IOP-Negativen vorlag, betrug dann $\mathrm{p}<0,001$. Damit ergab sich die Situation, dass sich nach arithmetischer Addition zweier Patientenkollektive eine hochsignifikante Irrtumswahrscheinlichkeit errechnen ließ, bei der die eigene Studie keine statistisch relevante Differenz in der Häufigkeitsverteilung des Merkmals typische Auslösesituation erkennen ließ, während in der anderen Studie ein signifikanter Unterschied angenommen werden konnte.

Mit prädiktiven Vorhersagewerten von jeweils $<70 \%$, Odds Ratios $<2,0$, Sensitivitäten mit einer Spannbreite von 33,3-61,9\% und Wahrscheinlichkeitswerten zwischen 0,77 und 0,06, die die Hypothese bestätigt hätten, dass es sich bzgl. des Auftretens einer typischen Auslösesituation zwischen den IOP-Positiven und -Negativen gehandelt hat, rechtfertigt unserer Meinung nach nicht, dass dieser Parameter ein valides Kriterium für die Indikationsstellung zur Durchführung einer explorativen Tympanoskopie darstellen kann, wie es auch schon Maier et al. hervorhoben hat [57].

Insgesamt hat man nach der von uns recherchierten Literatur mit einer Häufigkeit des Vorliegens charakteristischer Auslösesituationen in einem Hörsturzkollektiv zwischen 10,2 und 25,6\% zu rechnen [29, 75]. Auch andere Autoren hatten, ohne dies zahlenmäßig näher darzulegen, schon berichtet, dass keine konstante Beziehung zwischen dem Vorhandensein einer für eine PLF typischen Auslösesituation und dem späteren intraoperativen Nachweis einer PLF besteht $[29,97]$.

\subsection{Mittelohrbefunde und Schwindel}

Über Schwindel hatte einer von unseren 7 Patienten (14,3\%; entspricht prädiktivem Wert) mit auffälligen Paukenbefunden geklagt (Tab 7.3). Bei den Erkrankten mit unauffälligen Mittelohrstrukturen lag die Häufigkeit bei 22,0\% $(n=14 / 52)$. Es ließ sich eine Odds-Ratio von $\mathrm{OR}=1,9$ und ein Korrelationskoeffizient von $r=0,094$ berechnen.

Zwei weitere Autoren hatten eine vergleichbare Analyse ihres Datenmaterials vorgenommen [12, 57]. Bei Zusammenführung aller drei Patientengruppen lag man in 55,4\% der Fälle richtig (prädiktiver Wert) mit der Vorhersage einer PLF, wenn man sich auf das Symptom Schwindel bezog. Die aus allen Studien zusammenfasste, gemeinsame Odds Ratio wies einen Wert von 2,31 auf, was bedeutet, dass Schwindel genau um diesen Faktor häufiger bei Patienten mit pathologischen Mittelohrbefunden aufgetreten war als bei jenen, die unauffällige Paukenstrukturen zeigten. 
Tab. 7.3 Zusammenhänge zwischen IOP-positiven und IOP-negativen Befunden und dem Vorliegen von Schwindel im eigenen Patientengut (oberer Tabellenteil) sowie den von Denguine et al. [12], Maier et al. [57] publizierten Daten unter Einschluss des eigenen Patientengutes (unterer Tabellenteil)

\begin{tabular}{|c|c|c|}
\hline \multicolumn{3}{|c|}{ Eigenes Patientengut } \\
\hline & IOP path. & IOP о. B. \\
\hline Schwindel+ & 1 & 14 \\
\hline Schwindel- & 6 & 38 \\
\hline
\end{tabular}

Beziehung: Intratympanaler Befund - Schwindel

OR: 1,9

Prädiktiver Wert: 6,7\%

Sensitivität: $14,3 \%$

Spezifität: $73,1 \%$

$p=0,46$

Aus Studien zusammengefasstes Patientengut

\begin{tabular}{l|l|l}
\hline & IOP path. & IOP o. B. \\
\hline Schwindel+ & 56 & 45 \\
\hline Schwindel - & 43 & 80 \\
\hline
\end{tabular}

Beziehung: Intratympanaler Befund - Schwindel

OR: 2,31

Prädiktiver Wert: 55,4\%

Sensitivität: 56,6\%

Spezifität: $64 \%$

$p=0,002$

$I O P$ path.$=$ Patienten mit pathologischen Mittelohrbefunden; IOP $o . B .=$ Patienten mit normalen Paukenstrukturen; $O R=$ Odds Ratio; $p=$ Wahrscheinlichkeitswert, mit der sich beide Subgruppen von Patienten unterschieden

Bezüglich des Symptoms „Schwindel“ hatten sich bei dem Patientengut von Denguine et al., Maier et al. und unseren Patienten jeweils unterschiedliche Konstellationen in den Häufungen der IOP-positiven und IOP-negativen Patienten ergeben [12, 57]. Während bei uns nur 1 von 7 Patienten $(14,3 \%)$ mit pathologischem Mittelohrbefund eine Gleichgewichtsstörung aufwiesen, wurde dies bei Denguine et al. in 60,9 \% und bei Maier et al. in 59 \% der Fälle deutlich häufiger beobachtet (Sensitivität) $[12,57]$. Die Irrtumswahrscheinlichkeit, dass der Unterschied in der Häufigkeit des Auftretens von Schwindel bei den IOP-Positiven und IOP-Negativen zufällig war, lag bei $\mathrm{p}=0,002$. Dabei konnten Denguine et al. und wir in unserem Patientenkollektiv jeweils keinen statistisch signifikanten Hinweis ableiten, dass das Symptom einen Parameter darstellt, aufgrund dessen sich die Untergruppen der IOP-Positiven und -Negativen statistisch relevant unterscheiden lassen (Denguine et al.: $\mathrm{p}=0,44$; wir: $\mathrm{p}=0,46$ ). Lediglich Maier et al. gelang dieser Nachweis $(\mathrm{p}=0,005)$. Wenn zusätzlich prädiktive Werte von maximal $67 \%$ (Maier et al.) zu erreichen waren und die Odds Ratios zwischen 0,6 [12] und 2,8 [57] lagen, ziehen wir aus der Summe dieser Ergebnisse den Schluss, dass 
auch das Symptom Schwindel nicht als eindeutiger Indikator für das Bestehen bzw. Nichtbestehen einer Perilymphfistel gewertet werden kann. Damit mangelt es der gesamten präoperativen Diagnostik weiterhin an einem verlässlichen Parameter für die Vorhersage einer PLF [2, 4, 8, 37, 39, 70, 83, 105]. Man wird die Indikation zur Tympanoskopie wohl auch in der nahen Zukunft weiterhin nur von relativ ,vagen“ Vermutungen abhängig machen bzw. als Ultima Ratio nach anderen fehlgeschlagenen Behandlungsversuchen ansetzen.

\subsection{Zusammenhang Auslöser und Schwindel}

Schließlich interessierte noch, ob das Bestehen einer typischen Auslösesituation mit dem synchron zum Hörsturz aufgetreten Schwindel - beides als für PLT angegebene charakteristische Parameter - in einem direkten Zusammenhang steht. Die Tab. 7.4 zeigt, dass resultierend aus unseren Patientenbeobachtungen in den Fällen, in denen eine Auslösesituation eruiert werden konnte, zur Hälfte $(n=8)$ Schwindel vorlag, die anderen $50 \%$ hatten über das Symptom nicht geklagt. Umgekehrt lag, wenn keine Auslösesituation bestand, bei 45,2 \% der Erkrankten $(n=19)$ trotzdem ein Schwindel vor, in 54,8 \% der Fälle bestand keine Gleichgewichtsstörung. Der aus der Vier-Felder-Tafel eruierte Korrelationskoeffizient von $r=0,043$ und eine Odds Ratio von 1,2 zeigen, dass die beiden Parameter in keinem deutlichen Zusammenhang stehen.

\subsubsection{Hörentwicklung bei Patienten mit und ohne Perilymphfistel}

\subsubsection{Hörentwicklung (0,5, 1, 2 und 4 kHz) nach Tympanoskopie}

Wenn man für die Ermittlung des durchschnittlichen Hörverlustes die Frequenzen 0,5, 1, 2 und $4 \mathrm{kHz}$ verwendet, wiesen die von uns behandelten IOP-Positiven einen Hörverlust von anfangs 97,8 dB (SAW: 9,3 dB; $n=4)$ auf (Abb. 7.1). Dieser verbesserte sich nach der Operation um 47,2 dB (SAW: 23,3 dB), sodass der Hörlevel im Rahmen der Nachuntersuchung dann nur noch 50,7 dB (SAW: $31,9 \mathrm{~dB} ; \mathrm{n}=4$ ) betrug.

Tab. 7.4 Zusammenhang in der Häufigkeit des Auftretens eines zeitgleich mit der Hörminderung berichteten Schwindels und dem Vorliegen einer für eine PLF typischen Auslösesituation im eigenen Patientengut

\begin{tabular}{l|l|l|l}
\hline & Auslöser & Kein Auslöser \\
\hline Schwindel & 8 & 19 & 27 \\
\hline Kein Schwindel & 8 & 23 & 31 \\
\hline & 16 & 42 & 58 \\
\hline
\end{tabular}

Odds-Ratio: 1,2

Irrtumswahrscheinlichkeit: $p=0,72$ 


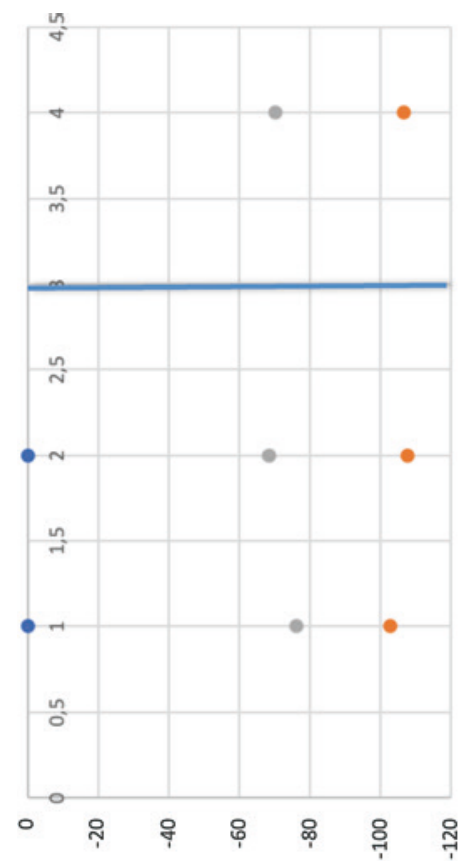

کิ

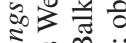
密 จิ से ริ 绻

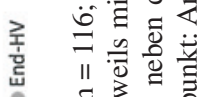

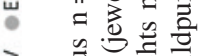

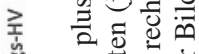

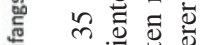
¿ II 氜苛 - चี 으류 Ð 跑 㐫 O) 으

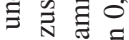

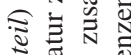

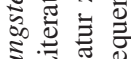

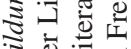
可:

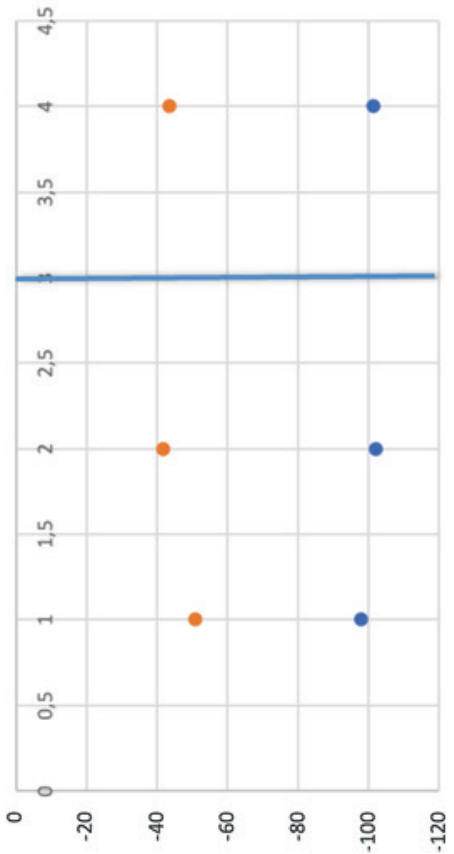
䒕包

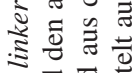

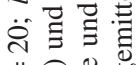
11 สิ = :

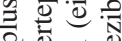

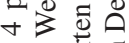
$>\quad I 1 \%$

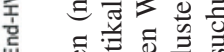

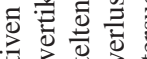

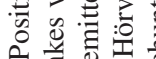

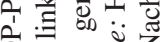

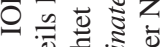

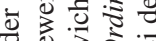

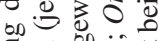

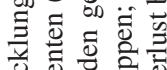

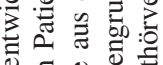

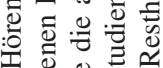
$-\frac{\infty}{0} \cdot \ddot{0}$ กั

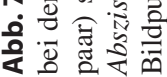


Die IOP-Negativen zeigten demgegenüber vor der Tympanoskopie einen mittleren Hörverlust von 102,7 dB (SAW: 17,2; $\mathrm{n}=35$ ) (Abb. 7.1). Der letzte gemessene Wert (2,3 Jahre nach dem Hörsturz) betrug dann 75,9 dB (SAW: 32,0 dB). Dem entspricht ein Hörgewinn von durchschnittlich 26,9 dB (SAW: 27,4 dB).

Keiner der 7 Patienten mit auffälligen Mittelohrbefunden hatte eine Surditas entwickelt, wohingegen die bei Patienten mit normalen Paukenstrukturen 21-mal der Fall war (30,4\% aller Fälle). Der Hörgewinn war in der Gruppe der IOPPositiven deutlich höher (Hörgewinn: 47,2 dB; SAW: 23,3 dB; $n=4$ ) als bei Patienten, die auch nach Paukeninspektion weiterhin als ,reine Hörstürze“ klassifiziert werden konnten (Hörgewinn: 26,9 dB; SAW: 23,3 dB; $n=35$ ).

Bei Thomas et al. wiesen die IOP-Positiven mit Anwendung der gleichen Auswertungsmethode einen Ausgangshörverlust von 101,9 dB auf (Abb. 7.1) [97]. Mit einer noch ausgeprägteren Hörerholung von $57,9 \mathrm{~dB}$, als wir sie in unserem Patientengut fanden, erreichten die Erkrankten dann bei der Followup-Untersuchung in dieser Studiengruppe einen Endhörwert von 43,3 dB. Im Vergleich dazu begannen die IOP- Negativen bei einem Hörlevel von 107,4 dB, zeigten dann eine Hörverbesserung von $37,4 \mathrm{~dB}$, um langfristig eine Hörminderung von $70,0 \mathrm{~dB}$ zurückzubehalten.

In einer aus beiden Patientenkollektiven zusammengefassten Analyse weisen die IOP-Positiven einen gewichteten Ausgangshörverlust von 106,4 dB auf, erzielten dann eine Hörerholung von 43,3 dB, um einen Endwert von 57,9 dB zu erreichen (Abb. 7.1, linker Abbildungsteil). Demgegenüber starteten die IOPNegativen mit einer anfänglichen Hörminderung von 107,4 dB. Sie verbesserten sich um durchschnittlich $36,4 \mathrm{~dB}$ und verblieben schließlich auf einem Hörlevel von $70,0 \mathrm{~dB}$.

\subsubsection{Gedankenexperiment: Schrittweise Hinzufügung anamnestischer Angaben bei der Diagnose einer Perilymphfistel}

\footnotetext{
Hintergrundinformation

Wenngleich bei IOP-negativen Patienten keine eindeutigen Zusammenhänge zwischen den Gleichgewichtsstörungen (Schwindel) und den für eine PLF typischen Auslösesituationen gefunden werden konnten (Tab.7.2 und 7.3), kommt diesen beiden Parametern doch eine gewisse Sonderrolle unter den von uns erhobenen patientenbezogen Daten zu, sind sie beide in der Literatur doch als charakteristische Begleitsymptome einer PLF beschrieben. In unserem Patientengut war, wie bereits oben beschrieben, die Quote intraoperativ entdeckter PLF mit 9,2 \% (9,5\%, wenn man sich nur auf Patienten bezieht, bei denen auch Langzeithörmessungen möglich waren) im Literaturvergleich ausgesprochen gering gewesen. Es ist deshalb nicht auszuschließen, dass wir bei der Tympanoskopie nicht alle der wirklich vorhandenen Leckagen zwischen dem Innen- und Mittelohr erkannt haben. Wir haben deshalb nachfolgend die gedankliche Simulation unternommen, in sukzessiven Einzelschritten die von Patienten mit einem Hörsturz vorgebrachten Angaben bei der Bewertung, ob eine PLF wahrscheinlich sein könnte oder nicht, mit zu berücksichtigen.

Man sieht in Abb. 7.2, dass in unserem Krankengut die mittleren Ausgangshörverluste bei den IOP-Positiven deutlich besser $(97,8 \mathrm{~dB})$ waren als bei den IOP-Negativen $(102,6 \mathrm{~dB})$. Bei der gedanklichen Simulation, dass wir von den IOP-Positiven $(n=4)$ ausgehend in einem ersten
} 
Schritt zusätzlich diejenigen Erkrankten mit in die Auswertung einbeziehen, bei denen zum Zeitpunkt des Hörsturzereignisses die für die Entstehung einer PLF charakteristische Auslösesituation bestanden hatte $(\mathrm{n}=10)$, und in einem weiteren Schritt von den IOP-Negativen noch diejenigen Erkrankten mit in die Analyse aufnahmen, die synchron zum Eintritt der Hörminderung über Schwindel geklagt hatten, entwickeln sich die Höhen des Ausgangshörverlustes, der Hörgewinne und damit auch der Endhörverluste schrittweise zu dem Ergebnis hin, wie wir es bei den „rein“ IOP-Negativen ohne Schwindelsymptomatik und ohne Bestehen der typischen Auslösesituation angetroffen hatten (Hinzufügung von Patienten mit typischen Auslösesituationen zu den ,nur“ IOP-Positiven: Anfangshörverlust: 110,2 dB; Hörgewinn: $35,3 \mathrm{~dB}$; Endhörverlust: 74,9 dB [n=14]; Hinzufügung von Patienten mit typischen Auslösesituationen und Schwindel zu den IOP-Positiven: Anfangshörverlust: 106,9 dB; Hörgewinn: 30,2 dB; Endhörverlust: 76,7 dB). Bei den IOP-Negativen (ohne typische Auslösesituation und ohne Schwindel) schließlich wurden folgende Werte gemessen: Anfangshörverlust: 87,2 dB; Hörgewinn: 32,0 dB; Endhörverlust: 55,2 dB. Der Ausgangshörverlust der IOP-Positiven fiel somit gegenüber dem der IOP-Negativen schlechter aus. Zugleich reduzierte sich der Hörgewinn sukzessive bei der schrittweisen Hinzufügung „positiver“ Patientenangaben und näherte sich damit auch dem der Patienten mit unauffälligen Mittelohrstrukturen bzw. ohne Vorliegen von charakteristischen Auslösesituationen und Gleichgewichtsstörungen schrittweise an. Bezogen auf die Endhörwerte konnten wir eine vergleichbare Beobachtung machen.

Es liegt nun an den Interpreten dieser Befunde, ob sie neben dem intraoperativen Befund auch zusätzlich die Patientenangaben mit in die Abschätzung der Häufigkeit vorliegender Perilymphfisteln in dem Kollektiv der Hörsturzpatienten einfließen lassen und damit sozusagen den Status des „Goldstandards“ des Tympanoskopieergebnisses schrittweise ,aufweichen“. Würde man die typischen Auslösesituationen vollwertig neben dem intraoperativen Befund als verbindliches Symptom für die Diagnose einer PLF gelten lassen, hätten wir einen Prozentsatz von 33,3 \% Innen-/Mittelohrleckagen in unserem Krankengut. Nähme man auch den Schwindel als einen weiteren Indikator einer PLF hinzu, käme man sogar auf einen Anteil von 71,4 \%.

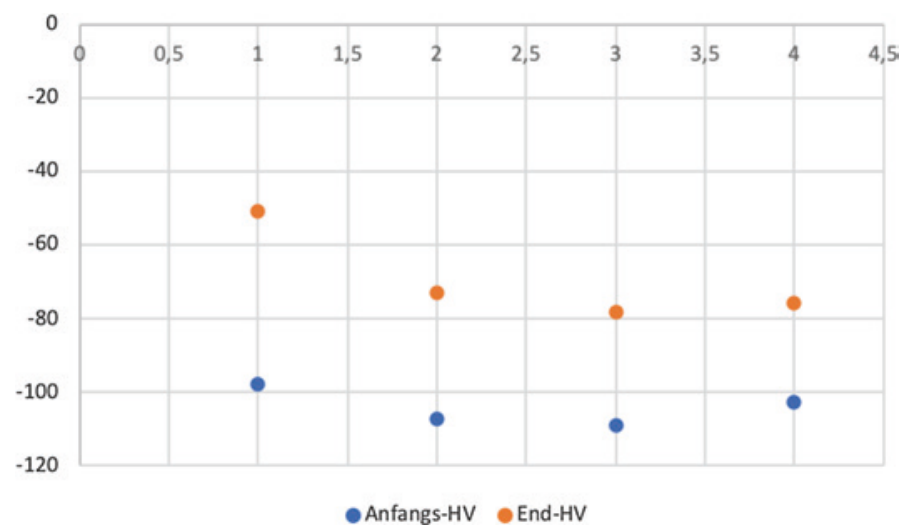

Abb. 7.2 Sukzessive Hinzunahme von Patientenangaben bei der Bewertung des Vorliegens einer PLF. Linkes vertikales Wertepaar: IOP-Positive (aufgrund des intraoperativen Befundes); links von der Mitte liegendes vertikales Wertepaar: IOP-Positive und Hinzufügung von Patienten mit zusätzlich einem der für PLF charakteristischen Befunde (typische Auslösesituation, Schwindel); rechts von der Mitte liegendes Wertepaar: IOP-Positive und Patienten, von denen zusätzlich 2 für eine PLF charakteristische Angaben vorgebracht worden waren (typischer Auslöser, Schwindel). Rechtes Wertepaar: IOP-Negative; Abszisse: Studiengruppen; Ordinate: Hörverluste in dB; unterer Bildpunkt: Anfangshörverlust; oberer Bildpunkt: Resthörverlust bei der Nachuntersuchung 


\subsubsection{Hörentwicklung $(0,25,1,4$ und $8 \mathrm{kHz})$ nach Tympanoskopie}

Legt man den gemittelten Hörverlust aus den vier Frequenzen $0,25 \mathrm{kHz}$, $1 \mathrm{kHz}, 4 \mathrm{kHz}$ und $8 \mathrm{kHz}$ in Anlehnung an die Auswertungsmethode von Maier et al. zugrunde, betrug der anfängliche Hörverlust bei den IOP-Positiven in unserem Patientengut 93,5 dB (SAW: 10,2; $\mathrm{n}=4$ ), der Endhörverlust 60,0 dB (SAW: 32,7 dB) (Abb. 7.3) [57]. Dem entsprach ein Hörgewinn von 33,5 dB (SAW: $26,9 \mathrm{~dB}$ ). Die IOP-Negativen hatten vor Therapiebeginn einen durchschnittlichen Hörverlust von 104,4 dB (SAW: 15,8 dB; $\mathrm{n}=32$ ). Im Rahmen der Nachuntersuchung wurde ein mittlerer Resthörverlust von 79,1 dB (SAW: 20,0 dB) gemessen, es war also zu einer Hörverbesserung um 25,3 dB (SAW: $7,0 \mathrm{~dB}$ ) gekommen.

Bei Maier et al. bestand bei den Patienten mit gesicherter PLF ein durchschnittlicher Ausgangshörverlust 90,2 dB (Abb. 7.3) [57]. Bei den langfristig verbliebenen Hörminderungen konnte ein gemittelter Endhörverlust von 60,1 dB dokumentiert werden, was mit einem Hörgewinn von 29,9 dB korrelierte.

Bei den IOP-Negativen kam es bei Maier et al. von einem durchschnittlichen initialen Hörverlust von 98,1 dB ausgehend zu einer Hörverbesserung von im Mittel 40,7 dB und zu einer langfristig verbleibenden, durchschnittlichen Hörminderung von 57,4 dB (Abb. 7.3) [57].

In einer aus beiden Populationen zusammengefassten Gruppe entwickelten sich die Hörverluste der IOP-Positiven von 90,3 dB ausgehend auf einen Wert von 60,1 dB, was mit einer Hörverbesserung von 30,2 dB einherging (Abb. 7.3).

Bei den IOP-Negativen lag der gewichtete Anfangshörverlust in unserem Krankengut bei 100,0 dB, der sich dann nach der Therapie auf einen Wert von 77,4 verbesserte, was mit einem durchschnittlichen Hörgewinn von 22,6 dB einherging (Abb. 7.3).

In der frequenzbezogenen Übersicht der Hörentwicklung sieht man, dass sich die Hörkurve bei den IOP-Positiven im eigenen Krankengut von einem relativ geringen Anfangshörverlust bei $0,25 \mathrm{kHz}$ von $76,3 \mathrm{~dB}$ (SAW: $28,7 \mathrm{~dB} ; \mathrm{n}=4$ ) in den darauffolgenden Tonhöhen mit Werten zwischen 95,0 dB (1 kHz) und 96,3 dB $(4 \mathrm{kHz})$ bis hin zu 106,3 dB $(8 \mathrm{kHz})$ eingestellt hatte, die alle recht nahe beieinander lagen (Abb. 7.4) [56]. Im Rahmen der Nachuntersuchung wurden in den einzelnen Frequenzen Hörverluste erreicht, die zwischen $30 \mathrm{~dB}(0,25 \mathrm{kHz}$; SAW: $26,8 \mathrm{~dB} ; \mathrm{n}=4)$ und $86,3 \mathrm{~dB}(8 \mathrm{kHz}$; SAW: $26,9 \mathrm{~dB} ; \mathrm{n}=4)$ betrugen. Der Kurvenverlauf zeigt einen nahezu linearen Abfall der Hörkurve von den tiefen zu den hohen Frequenzen (1 kHz: $50 \mathrm{~dB}$; 4 kHz: 73,8 dB).

Die IOP-Negativen wiesen in unserem Patientengut vor Therapiebeginn bei $0,25 \mathrm{kHz}$ einen Hörverlust von 102,0 dB (SAW: 22,0 $\mathrm{n}=34$ ) auf, der sich dann über 104,4 $(1 \mathrm{kHz})$ und $102,7 \mathrm{~dB}$ bis auf $108,4 \mathrm{~dB}(8 \mathrm{kHz}$; SAW: $15,8 \mathrm{~dB})$ erstreckte (Abb. 7.4). Bei der Nachuntersuchung wurden folgende Werte gemessen: 0,25 kHz 65,5 dB (SAW: 37,2 dB), $1 \mathrm{kHz} 73,3 \mathrm{~dB}, 4 \mathrm{kHz} 84,7 \mathrm{~dB}$ und $8 \mathrm{kHz}$ 93,0 dB (SAW: 23,1 dB). 


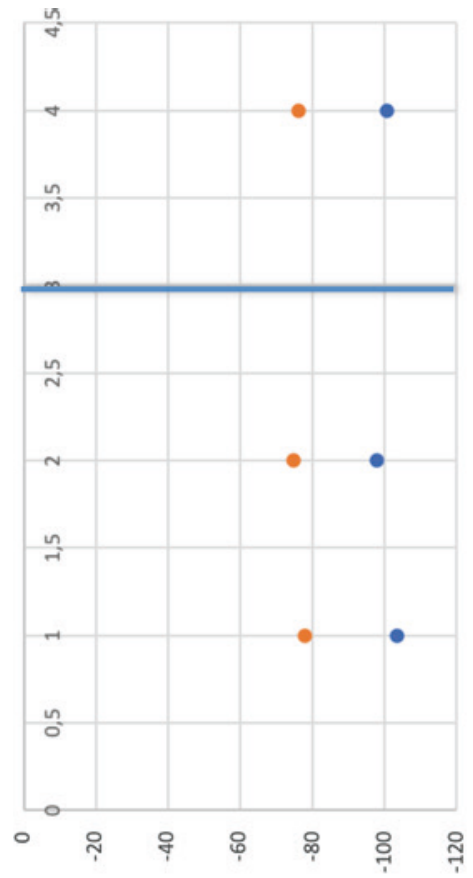

$\Xi$ 충

종

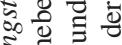

농

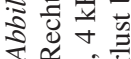

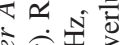

宗宣

证

过声产造

ج

II

들 흘 흠

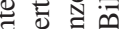

인

呵

б

至

品

\&

응

สं

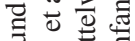

․․ㄹ

ปัँ

흥

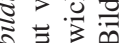

政

భ

赔

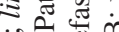

ळे

浔.

$\Xi$

5

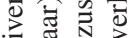

के

을

iे

의

牙新施

प

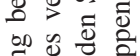

目娄壾

는

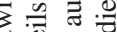

政

: 0

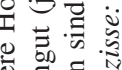

过

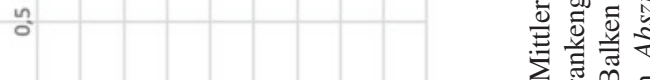

$\sum$ 㱐运

$m$.

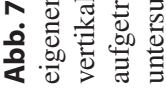

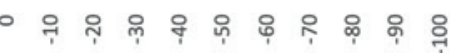




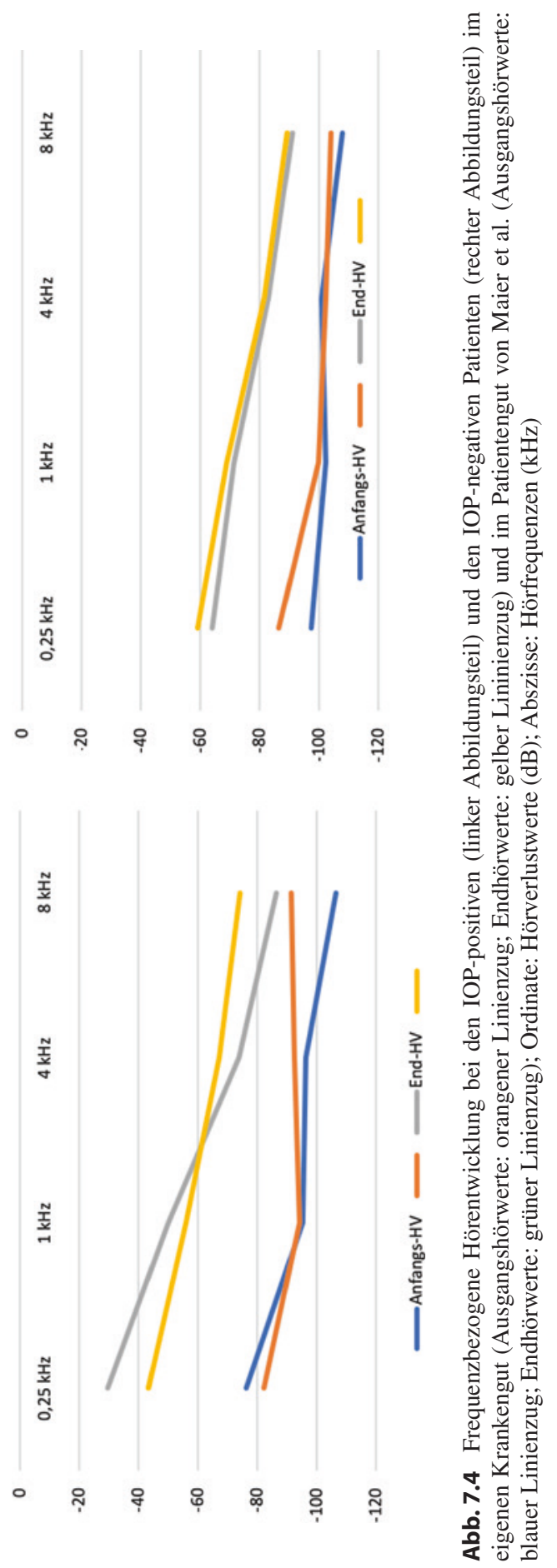


Bei Maier et al. lagen die Anfangshörverluste der IOP-positiven Patienten bei $82,1 \mathrm{~dB}(0,25 \mathrm{kH}), 94,1 \mathrm{~dB}(1 \mathrm{kHz}), 92,4 \mathrm{~dB}(4 \mathrm{kHz})$ und 91,2 dB (8 kHz) [57]. Die letzten gemessenen Hördaten im Rahmen der Nachuntersuchung betrugen $43,1 \mathrm{~dB}(0,25 \mathrm{kHz}), 55,7 \mathrm{~dB}(2 \mathrm{kHz}), 67,5 \mathrm{~dB}(4 \mathrm{kHz})$ und 74,1 dB $(8 \mathrm{kHz})$.

Die IOP-Negativen zeigten initiale Hörverluste von $86,5 \mathrm{~dB}(0,25 \mathrm{kHz})$, 99,8 dB (1 kHz), 102,2 dB (4 kHz) und 103,8 dB (8 kHz). Als Endhörwerte wurden angegeben $58,9 \mathrm{~dB}(0,25 \mathrm{kHz}), 68,8 \mathrm{~dB}(1 \mathrm{kHz}), 81,5 \mathrm{~dB}(4 \mathrm{kHz})$ und $89,3 \mathrm{~dB}(98 \mathrm{kHz})$.

Bei der Betrachtung der gewichtet gemittelten Hörkurverläufe lagen die Anfangshörverluste der IOP-Positiven zwischen $81,5 \mathrm{~dB}(0,25 \mathrm{kHz})$ und 106,3 dB $(8 \mathrm{kHz})$ (Abb. 7.5). Die Werte in den Frequenzen dazwischen betrugen bei $1 \mathrm{kHz}$ 94,2 dB und bei $4 \mathrm{kHz} 92,8 \mathrm{~dB}$. Im Rahmen der Nachuntersuchung wurden noch Höreinbußen von $41,7 \mathrm{~dB}$ bei $0,25 \mathrm{kHz}, 55,1 \mathrm{~dB}$ bei $1 \mathrm{kHz}, 68,2$ bei $4 \mathrm{kHz}$ und $75,4 \mathrm{~dB}$ gemessen.

Bei den initialen Hörverlusten wiesen die IOP-Negativen sich nur geringgradig von den IOP-Positiven unterscheidende Hördaten auf. Hier lagen die Anfangshörverluste für $0,25 \mathrm{kH}$ bei $93,8 \mathrm{~dB}$, für $1 \mathrm{kHz}$ bei $96,1 \mathrm{~dB}$, für $4 \mathrm{kHz}$ bei $102,4 \mathrm{~dB}$ und für $8 \mathrm{kHz}$ bei $106 \mathrm{~dB}$. Die Endhörverluste betrugen $62 \mathrm{~dB}(0,25 \mathrm{kHz})$, $70,9 \mathrm{~dB}(1 \mathrm{kHz}), 82,9 \mathrm{~dB}(4 \mathrm{kHz})$ und $91 \mathrm{~dB}(8 \mathrm{kHz})$.

Die Betrachtung der Hörkurvenverläufe deckte in beiden Studienkollektiven auf, dass im Tieftonbereich weitaus bessere Hörgewinne eingetreten waren, als sie in den hohen Frequenzen beobachtet werden konnten [56]. Hier erwiesen sich im eigenen Patientengut die Unterschiede in den Hörverlusten, die im Rahmen der Nachbeobachtung registriert wurden, in den Frequenzen $0,5 \mathrm{kHz}(\mathrm{p}=0,005)$ und $1 \mathrm{kHz}(\mathrm{p}=0,003)$ bei den IOP-Positiven sogar als signifikant besser, als sie sich bei den IOP-Negativen langfristig eingestellt hatten. Darüber hinaus ging aus den Kurven der Abb. 7.5 eindeutig hervor, dass die IOP-Positiven ausgeprägtere Hörgewinne erzielten, als es in der Gruppe der IOP-Negativen der Fall war. Schließlich ging aus den frequenzbezogenen Hörkurven noch hervor, dass von den tiefen zu den hohen Tonhöhen ein nahezu geradliniger Abfall der Hörwerte erfolgte, der bei den Ausgangsuntersuchungen geringer ausgeprägt war als bei den in der Nachuntersuchung ermittelten Hördaten.

\subsubsection{Hörentwicklung anhand des Kriteriums,$\geq 20 \mathrm{~dB}$ Hörgewinn" nach der Tympanoskopie}

In unserem Krankengut erreichten $75 \%$ der IOP-Positiven $(n=3)$ einen Hörgewinn von $\geq 20 \mathrm{~dB}$. Für die IOP-Negativen lag der Wert bei 62,8 \% (Abb. 7.6).

Haubner et al. berichten, dass in ihrem Kollektiv 53,8\% der Patienten mit einer PLF eine Hörverbesserung von $\geq 20 \mathrm{~dB}$ erzielten, während dies bei den Erkrankten mit normalen Mittelohrbefunden nur in 58,8\% der Fälle zutraf (Abb 7.5) [29].

Fasst man beide Populationen im Rahmen einer gewichteten Mittelwertbildung zusammen, erreichten 58,8 \% der IOP-Positiven einen Hörgewinn von $\geq 20 \mathrm{~dB}$. Dem stehen 61,2\% der Patienten gegenüber, die keinen auffälligen Mittelohrbefund boten (Abb. 7.6). 


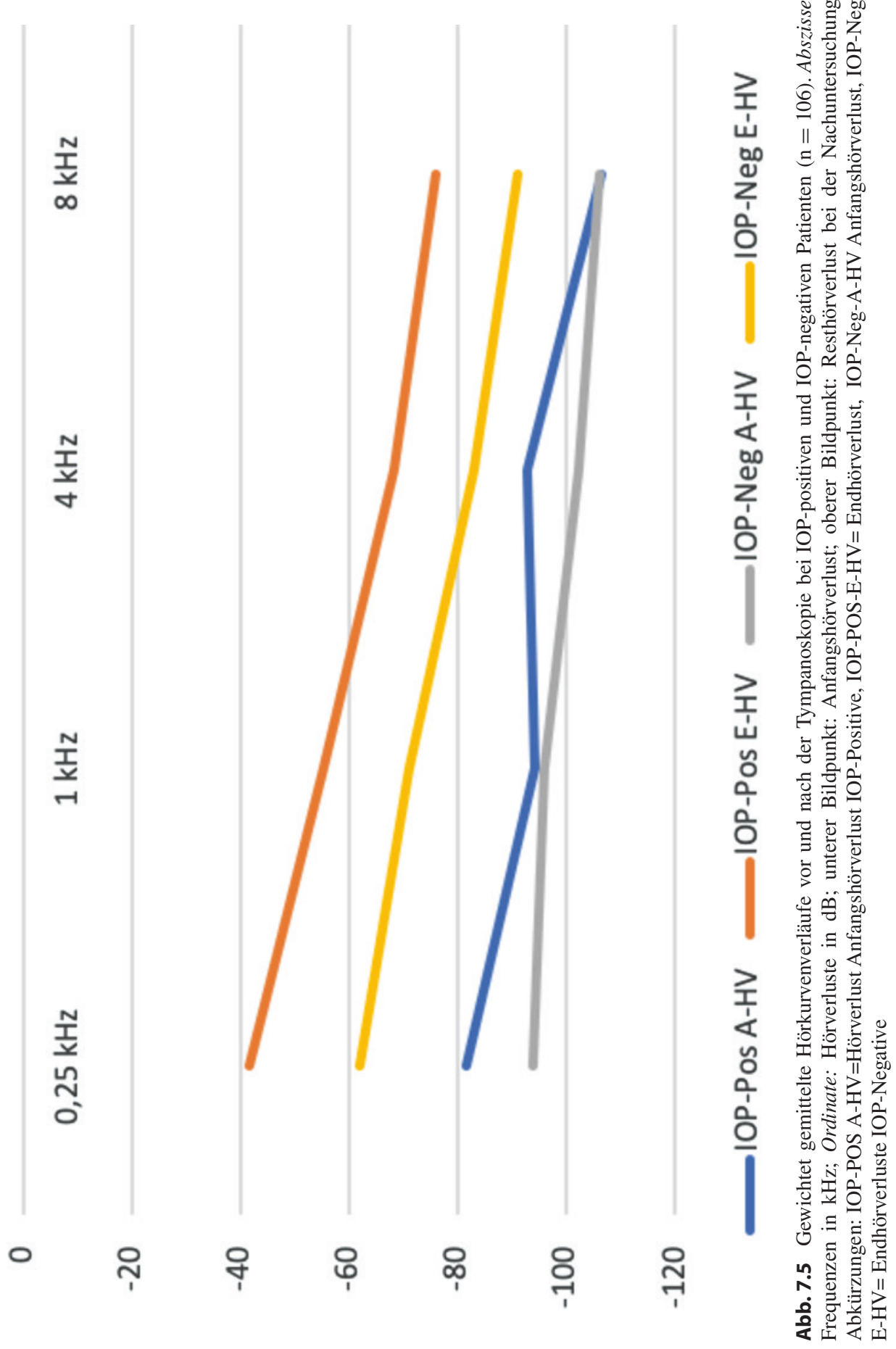




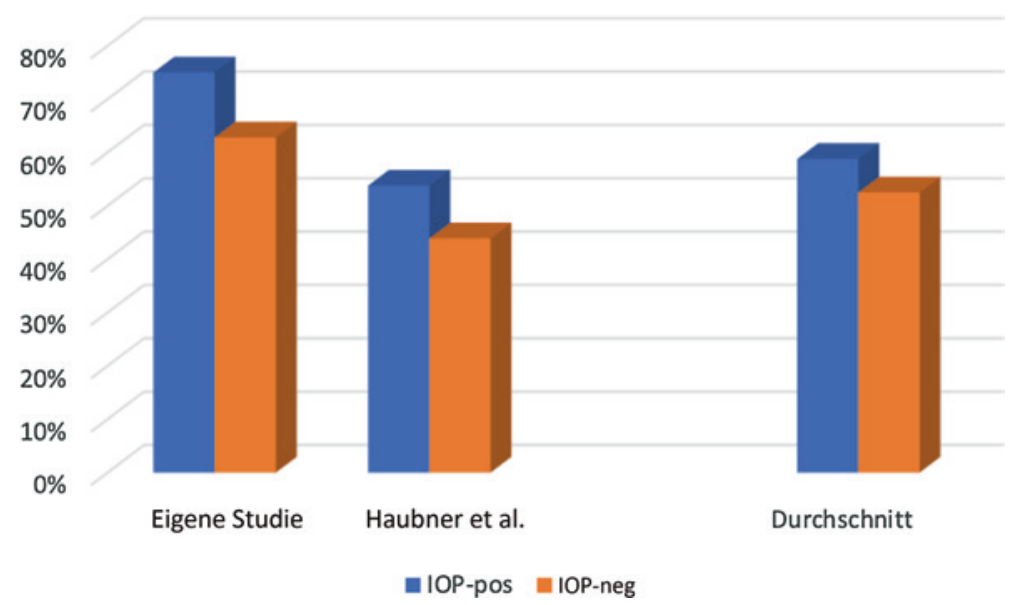

\begin{abstract}
Abb. 7.6 Hörgewinne bei den IOP-positiven (jeweils linker Balken eines Balkenpaares) und IOP-negativen Patienten (jeweils rechter Balken eines Balkenpaares; $n=52$ ) im eigenen Krankengut (linkes Balkenpaar) und im Patientengut von Haubner et al. [29] (mittleres Balkenpaar) und die aus beiden Studiengruppen zusammengefassten gewichteten Mittelwerte (rechtes Balkenpaar). Hörgewinne $\geq 20 \mathrm{~dB}(\mathrm{n}=17)$ vs. $<20 \mathrm{~dB}(\mathrm{n}=52)$
\end{abstract}

Bei dieser Art der Beurteilung der Hörgewinne blieb der bislang eindeutige Unterschied mit der Darstellung günstigerer Hörerholungen bei Patienten mit auffälligen Mittelohrbefunden gegenüber jenen, die normale Paukenstrukturen besaßen, weitgehend aus.

\title{
7.2.2.5 Hörentwicklung anhand der prozentualen Hörverluste (gemäß Bönninghaus) nach Tympanoskopie
}

Dem Algorithmus von Bönninghaus et al. folgend, der sich ebenfalls auf die Frequenzen $0,5,1,2$ und $4 \mathrm{kHz}$ bezog und bei dem prozentuale Hörverluste angegeben wurden, lag der Anfangshörverlust der IOP-positiven Patienten in unserem Krankengut bei einem $98 \%$ igen anfänglichen Hörverlust $(n=4)$ (Abb. 7.7). Bei der Nachbeobachtung wurde noch ein Hörverlust von $52 \%$ ermittelt. Dem entspricht eine Hörverbesserung von $46 \%$. Die IOP-Negativen lagen mit ihren Höreinbußen anfangs ebenfalls bei $98 \%(n=35)$. Im Rahmen des Nachuntersuchungstermins wurde in diesem Patientengut noch ein mittlerer Hörverlust von 62,4 \% eruiert. Es wurde ein Hörgewinn von 35,6 \% erreicht.

In der Studiengruppe von Prenzler et al. bestand vor Therapiebeginn ein prozentualer Hörverlust von $88 \%$ bei den IOP-Positiven $(n=16)$. Langfristig lag dann nur noch eine Höreinbuße von $65 \%$ vor (Abb. 7.7). Dem entspricht eine prozentuale mittlere Hörverbesserung von $23 \%$. Die IOP-Negativen $(n=41)$ wiesen zu Beginn einen geringgradig höheren mittleren Hörverlust von $94 \%$ auf, der sich nach der Therapie inkl. der Nachbeobachtungszeit auf $77 \%$ reduziert hatte. Der Hörgewinn betrug in dieser Gruppe der Erkrankten $17 \%$. 


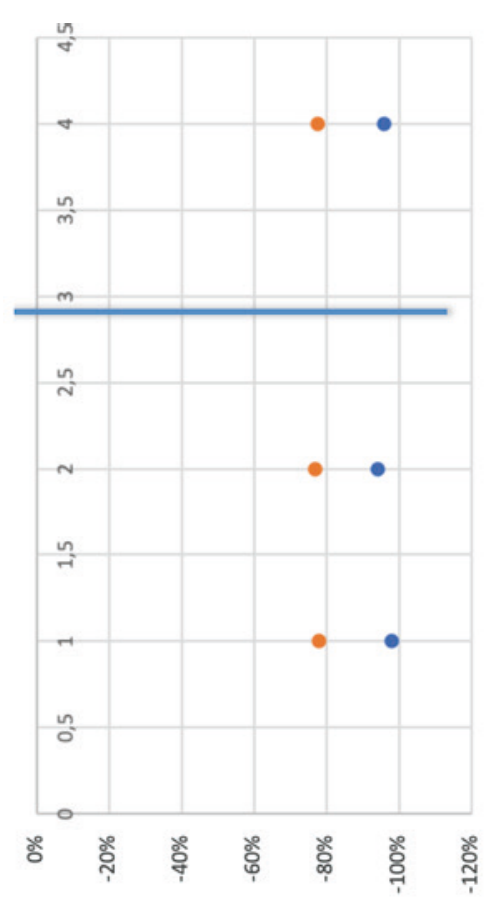

紊离

iิ $\frac{0}{\pi} \ddot{d}$

$\|$ 要要

$\Xi$

ब्ष

氖 卷

ब.

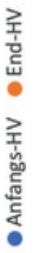

능

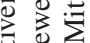

证

तं

너웡

을

可

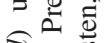

胥

范?

苛

ङ

《.

范

ミョ

穴司焉

$\|$ ए

ङ

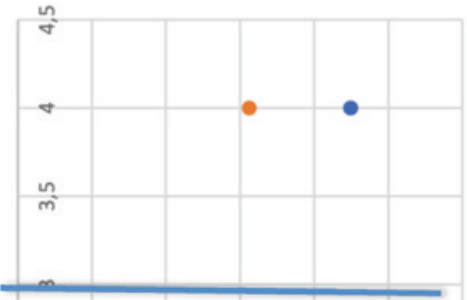

ฮี 产的 总离 के 8

으를

궁 응

흥 $>0$

ब.

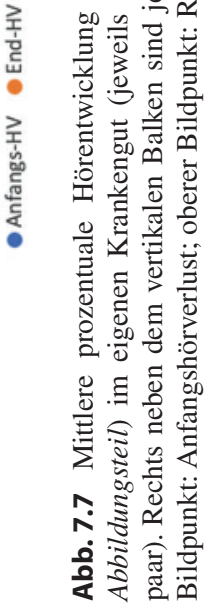


Das gewichtete Mittel des Ausgangshörwertes beider IOP-positiven Patientenkollektive lag bei $90 \%(n=20)$ (Abb. 7.7). Mit einer durchschnittlichen Hörverbesserung von 27,6\% erreichten sie einen Endwert von 62,4\%. Demgegenüber begannen die IOP-negativen Patienten im zusammengefassten Patientengut mit einem Hörverlust von 95,8 \% $(n=52)$. Sie konnten durch einen Hörgewinn von im Mittel 18,3 \% einen prozentualen Endhörverlust von 77,5 \% bei der Follow-upKontrolle erreichen.

\subsubsection{Bewertung der Ergebnisse - ungleiche Auswertungsmethoden}

Wie bereits an anderer Stelle aufgeführt, ist es bedauerlich, dass die vier zur Auswertung der Hörentwicklung nach Tympanoskopie geeigneten Studien jeweils unterschiedliche Verfahren zur Bemessung des Therapieergebnisses eingesetzt haben. Dies ließ zusammen mit den von uns Behandelten leider immer nur einen Vergleich mit einer weiteren Patientenpopulation zu. Auf diese Weise konnten keine auf einem größeren Kollektiv basierende Aussagen erarbeitet werden. Gerade solche wären aber notwendig, um die Ergebnisse der Tympanoskopie mit Abdeckung der runden/ovalen Fensternische auf einem statistisch abgesicherten Niveau herstellen zu können. Trotzdem ließen sich bei der Analyse der einzelnen Untersuchungen in der vorliegenden Form deutlich übereinstimmende Trends erkennen.

Bei der Auswertung der Hörentwicklung nach explorativer Tympanoskopie bei den Patienten mit und ohne Perilymphfisteln stießen wir auf ein sich mehrfach wiederholendes Phänomen. Sowohl, wenn die Hörverbesserungen mithilfe der PTA $_{4}$ aus den Frequenzen 0,5, 1, 2 und $4 \mathrm{kHz}$ gebildet wurden, als auch, wenn die Frequenzen 0,25, 1, 4 und $8 \mathrm{kHz}$ zur Bildung eines Durchschnittswertes herangezogen wurden, zeigte sich, dass von nahezu gleich hohen Anfangshöreinschränkungen in den verschiedenen Studienkollektiven, einschließlich des unsrigen, die Erkrankten mit pathologischen Mittelohrbefunden zum Teil weitaus ausgeprägtere Hörerholungen erreichten als die IOPNegativen [57, 97]. Auch wenn das Selektionskriterium , $\geq 20 \mathrm{~dB}$ Hörgewinn“ vs. „<20 dB Hörgewinn“ zur Analyse der Hörentwicklung herangezogen wurde, entstand ein ähnliches Bild. Haubner et al. konnten geringgradig bessere Hörverbesserungen bei den Patienten mit PLF registrieren als bei Patienten, die normale Paukenstrukturen aufwiesen [29]. Selbst bei Verwendung der prozentualen Hörverluste, ermittelt aus den Frequenzen 0,5, 1, 2 und $4 \mathrm{kHz}$, machten Prenzler et al. eine zu den bisherigen Ergebnissen identische Beobachtung. Patienten mit PLF erreichten nach dem chirurgischen Eingriff eine durchschnittlich bessere Hörverbesserung als Patienten mit unauffälligem Mittelohr [75].

Diese in allen Studiengruppen übereinstimmenden Ergebnisse sind möglicherweise ein Ausdruck dafür, dass bei Patienten mit Perilymphfisteln die Abdeckung der runden, teilweise zusätzlich auch der ovalen Fensternische, eine kausale Behandlungsmethode darstellt, die das Leck zwischen dem Innen- und Mittelohr wirksam abdichten kann, und auf diese Weise in einem hohen Prozentsatz der Fälle zum Erfolg geführt hat. Dennoch und das ist erstaunlich, können 
auch die IOP-Negativen mit diesem Therapieverfahren, bei denen eine vergleichbare Erklärung für die physiologische Wirkung der operativen Maßnahme Hyperämien, die aufgrund verschiedener Erholungsmechanismen im Innenohr im Bereich der runden/ovalen Fensternische induziert werden - bis auf Weiteres recht vage bleibt, zu recht respektablen Ergebnissen gelangen [42]. Selbst wenn in mehreren Subpopulationen kein signifikanter Vorteil der Patienten mit PLF gegenüber den IOP-Negativen bezüglich der Hörverbesserungen in der Literatur nachgewiesen werden konnte, scheint in der Zusammenschau der hier präsentierten Daten die Wahrscheinlichkeit doch naheliegend, dass die Erkrankten mit Perilymphfisteln mehr von einer explorativen Tympanoskopie mit Obliteration der runden/ovalen Fensternische profitieren als diejenigen, bei denen das Perilymphleck nicht so eindeutig nachweisbar ist [97].

Maier et al. leiteten aus dieser Beobachtung ab, auch im Hinblick des äußerst geringen Komplikationsrisiko, mit dem der chirurgische Eingriff einhergeht, dass eine großzügige Indikationsstellung zur explorativen Tympanoskopie mit Obliteration der runden/ovalen Fensternische gerechtfertigt erscheint. Mit dieser Art des Vorgehens werden IOP-Positive in einem hohen Prozentsatz der Fälle positiv beeinflusst und auch für IOP-Negative scheint sich diese Maßnahme, wenn überhaupt, kaum ungünstig auf die weitere postoperative Hörentwicklung auszuwirken [57].

Auch wenn unser eigenes Patientengut mit auffälligen Mittelohrbefunden, die auch in der Verlaufsbeobachtung zu beurteilen waren, nur aus 4 Einzelfällen bestand, standen die von uns erzielten Resultate in einer erstaunlichen Übereinstimmung mit denen aus zahlenmäßig umfangreicheren Patientenkollektiven anderer Studiengruppen. Insofern ist auch unserem Beitrag zu der Gesamtauswertung eine gewisse Validität zuzubilligen.

\subsection{Zusammenfassung}

Eine explorative Tympanoskopie, die bei Patienten mit einer ISSNHL durchgeführt wird, deckt in einer Reihe von Fällen das Bestehen einer Perilymphfistel (PLF) auf. Der vorliegende Beitrag geht einerseits der Frage nach, inwieweit sich präoperativ ein derartiges Leck zwischen dem Innen- und Mittelohr voraussagen lässt, und andererseits ob Patienten mit pathologischem Mittelohrbefund (IOPPositive) eine von den sonstigen Hörsturzpatienten (IOP-Negative) abweichende Hörentwicklung nach dem operativen Eingriff aufweisen. In die Auswertung konnten aus dem eigenen Krankengut 51 Patienten und aus vier veröffentlichten Studien zusätzlich 317 Erkrankte einbezogen werden.

Im eigenen Patientengut zeigte sich, dass die im Rahmen der Klinikaufnahme erhobenen epidemiologischen Daten, anamnestischen Beschwerden und klinischen Erstbefunde keine signifikant unterschiedlichen Häufigkeitsverteilungen unter den IOP-Positiven und IOP-Negativen ergaben.

Bezüglich des Vorliegens einer für die PLF typischen Auslösesituation beträgt der aus der Studie von Maier et al. sowie unserem Krankengut $(n=193)$ 
zusammengefasste prädiktive Wert 30,2 \% und die 5-\%-Irrtumswahrscheinlichkeit für den Nachweis einer signifikant unterschiedlichen Verteilung zwischen den IOP-Positiven und IOP-Negativen $\mathrm{p}=0,0008$.

Die entsprechenden Werte betragen für den Schwindel - unter zusätzlicher Hinzufügung der Analysen von Denguine et al. (Gesamtpatientenzahl: n=224) $55,4 \%$ (prädiktiver Wert) und $\mathrm{p}=0,002$.

Die sich zum Teil widersprechenden Aussagen der aus den zusammengelegten Patientenkollektiven berechneten Daten über die Zuverlässigkeit typischer Auslösesituationen und des Schwindels im Hinblick auf die exakte Prognose einer PLF reflektieren dabei aber nicht die Aussagen, die aus der Interpretation der jeweiligen Einzelstudien möglich gewesen wären. Insofern glauben wir, dass diese Parameter keinen ausreichenden Hintergrund für die Indikation zur Tympanoskopie liefern können.

Unabhängig von der Art der Berechnung der Hörgewinne konnten nach der Tympanoskopie jeweils bessere Hörresultate für die IOP-Positiven gegenüber den IOP-Negativen erreicht werden. Bei den Frequenzen $0,5 \mathrm{kHz}(\mathrm{p}=0,05)$ und $1 \mathrm{kHz}$ ( $\mathrm{p}=0,033)$ ergaben sich bei der Nachuntersuchung (2,2 Jahre nach dem Hörsturzereignis) im eigenen Patientengut signifikant günstigere, langfristig bestehend bleibende, durchschnittliche Hörverluste für die Patienten mit auffälligen Mittelohrbefunden gegenüber jenen mit normalen Paukenstrukturen. Auch die IOPNegativen erreichten noch beachtliche Hörerholungen nach dem operativen Eingriff.

Würde die Diagnose einer PLF nicht nur von den intraoperativ erhobenen Paukenbefunden abhängig gemacht, sondern auch bei einem Teil derjenigen Patienten angenommen, bei denen ein Schwindel zum Zeitpunkt des Hörsturzereignisse aufgetreten war oder eine für die Entstehung einer PLF typische Auslösesituation bestand, würde sich die Anzahl der Patienten mit Perilymphleck erhöhen. Damit würde sich die Hörentwicklung nach dem operativen Eingriff zunehmend an diejenige angleichen, die die Erkrankten mit regelrechten Paukenstrukturen aufgewiesen hatten, d. h., die geringeren Anfangshörverluste und ausgeprägteren Hörerfolge der IOP-Positiven würden sich schrittweise den schlechteren Ausgangshörwerten und geringeren Hörerholungen der IOPNegativen annähern. 
Open Access Dieses Kapitel wird unter der Creative Commons Namensnennung 4.0 International Lizenz (http://creativecommons.org/licenses/by/4.0/deed.de) veröffentlicht, welche die Nutzung, Vervielfältigung, Bearbeitung, Verbreitung und Wiedergabe in jeglichem Medium und Format erlaubt, sofern Sie den/die ursprünglichen Autor(en) und die Quelle ordnungsgemäß nennen, einen Link zur Creative Commons Lizenz beifügen und angeben, ob Änderungen vorgenommen wurden.

Die in diesem Kapitel enthaltenen Bilder und sonstiges Drittmaterial unterliegen ebenfalls der genannten Creative Commons Lizenz, sofern sich aus der Abbildungslegende nichts anderes ergibt. Sofern das betreffende Material nicht unter der genannten Creative Commons Lizenz steht und die betreffende Handlung nicht nach gesetzlichen Vorschriften erlaubt ist, ist für die oben aufgeführten Weiterverwendungen des Materials die Einwilligung des jeweiligen Rechteinhabers einzuholen.

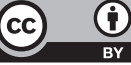

